\title{
The Latin Gerund and Its Translation in Albanian
}

\author{
Dr. Leonard Xhamani \\ Docente di Latino, Università di Tirana \\ Email: leonardxhamani@gmail.com
}

\section{Doi:10.5901/mjss.2014.v5n20p2332}

\section{Abstract}

The Latin language continues to be an object of study in order to enable the achievement of consolidated cultural knowledge of the cultural European roots. Reading and translating the Latin authors creates the opportunity to get into the antique culture heritage and indirectly in all the cultures that are expressed in the Latin language. In order to achieve a difficult objective, such as the interlingual and cross cultural confrontation, translation is helped by the comparative linguistics through pointing out the different grammatical aspects of different languages. In the following article, which belongs to this field of study, we have analyzed the gerund of the Latin language, a verbal non-finite form which does not have only one respective form in Albanian. We have examined the way this non-form is created, the different characteristics it has (nominative, verbal), the cases that is used in, the groups of the verbs which have no gerund form, the various morphological functions, the syntactic links it establishes with other parts of the speech, etc. In all the stages of the analyses, the corpus of the examples translated in Albanian illustrates and makes this special non-finite form of the Latin language quite perceivable even for Albanian speakers.

Keywords: Albanian language, gerund, Latin language, non-finite form, translation.

Il gerundio latino, pur appartenendo al sistema verbale, detiene le caratteristiche del sostantivo del genere neutro. Esso viene definito sostantivo verbale che designa un'azione in quanto processo. Nel gerundio si intrecciano, dunque, caratteristiche verbali e nominali. Per quanto riguarda la definizione, possimo dire che il gerundio latino assomiglia al gerundio albanese, esprime un'azione che accompagna l'azione del verbo reggente, con la quale si compie di solito contemporaneamente. II gerundio albanese viene caratterizzato, però, da caratteristiche verbali ed avverbiali. II gerundio si forma in modo sintetico, aggiungendo al tema del presente il suffisso -nd, per i verbi di prima e seconda coniugazione ed il suffisso -end per i verbi della terza e quarta coniugazione, nonché le flessioni nominali della seconda declinazione (Tantucci, 1992: 117). Dalla modalità di formazione, il gerundio assomiglia al participio che si forma con il suffisso -ens (ent-), (vole-ns - volent-is) (Alfred Ernout \& François Thomas, 1964: 263). Vediamo di seguito come si forma il gerundio per le quattro coniugazioni:

$\begin{array}{llll}\text { Coniug. I } & \text { II } & \text { III } & \text { IV } \\ \text { Gen. lauda-nd-i } & \text { mone-nd-i } & \text { leg-end-i } & \text { audi-end-i } \\ \text { Dat. lauda-nd-o } & \text { mone-nd-o } & \text { leg-end-o } & \text { audi-end-o } \\ \text { Acc. ad lauda-nd-um } & \text { ad mone-nd-um } & \text { ad leg-end-um } & \text { ad audi-end-um } \\ \text { Ab1. lauda-nd-o } & \text { mone-nd-o } & \text { leg-end-o } & \text { audi-end-o }\end{array}$

$>$ Milites flagrabant cupiditatae pugnandi. - Ushtarët vlonin nga dëshira e të luftuarit (për të luftuar). (Kosta Qiriazati \& Roza Dishnica, 1989: 117)

$>$ Milites intenti erant pugnando. - Ushtarët ishin të gatshëm për të luftuar.

$>$ Dux milites ad pugnandum confirmavit. - Komandanti u dha zemër ushtarëve të luftonin.

$>$ Milites fortifer pugnando urbem ceperunt. - Ushtarët duke luftuar me trimëri e morën qytetin.

II termine gerundio proviene dal verbo gerĕre ed indica ciò che dev'essere fatto. Si usa solamente nella forma attiva e si forma in quasi tutti i verbi attivi o quelli deponenti quali: hortandi (da hortari - nxis), verendi (da vereri frikësohem), sequendi (da sequi - ndjek), largiendi (da largiri - dhuroj), transitivi ed intransitivi (Terracina, 2003: 68). Esistono, però, anche verbi che non hanno la forma del gerundio quali: sum (jam), possum (mund), queo (mundem), nequeo (nuk mundem), aio (them), alcuni verbi impersonali quali: decet (më përshtatet), libet (më pëlqen), licet (lejohet), ecc. Il gerundio non ha la categoria del tempo, ma ha quattro casi, come abbiamo avuto modo di notare precedentemente (il genitivo, il dativo, l'accusativo e l'ablativo). Nella lingua latina il gerundio si avvicina semanticamente all'infinito, poiché si considera come complementare del sistema nominale di quest'ultima forma quando si usa con la funzione di un sostantivo. L'infinito si usa nella funzione del soggetto e del complemento oggetto, mentre il gerundio si 
usa nelle funzioni dei casi irregolari dell'infinito (Piazzino \& Fragonara, 1985: 420). Comunque sia le caratteristiche nominali del gerundio sono più accentuate rispetto a quelle dell'infinito. Questo viene confermato anche dalla seguente tabella.

Nominativo - Tacēre est necessarium - E heshtura (të heshtësh) është e nevojshme.

Genitivo - Tacendi necessitas - Nevoja e të heshturit (e heshtjes).

Dativo - Tacendo operam do - Do të angazhohem të hesht (në heshtje).

Accusativo - Ad tacendum vir natus - Njeri i lindur për të heshtur (të heshtë).

Ablativo - Tacendo multa consequēris - Duke heshtur (me heshtjen) do të fitosh shumë.

2s N.B. (Tantucci, 1992: 367)

L'accusativo è sempre unito con una preposizione e si traduce con un complemento indiretto; come complemento diretto si usa l'infinito semplice. Per esempio: Cupio legěre - Dëshiroj të lexoj.

II gerundio ha sempre significato attivo e quando si usa nella funzione del sostantivo, si mette nel caso richiesto dal verbo. Per chiarire meglio, nella seguente tabella diamo l'uso nominale del gerundio a seconda dei casi e della modalità di sostituzione da un sostantivo in questi casi. A mo' d'esempio è stato preso il gerundio del verbo studēre (studioj), il quale viene sostuito dal sostantivo studium (studim) (Basha, 1974: 33).

Gen. Tempus studendi - koha e të studiuarit Tempus studii $\quad$ - koha e studimit

Dat .Do operam studendo - përpiqem për të studiuar (të studioj) Do operam studio - përpiqem për studim

Ac. Eo ad studendum - shkoj për të studiuar Eo ad studium - shkoj për studim

Abl.Discitur studendo - mësohet duke studiuar Discitur studio - mësohet me anën e studimit

Come si può notare, il gerundio del latino fa le veci del sostantivo, poiché si declina come un sostantivo, se fosse sostituito da quest'ultimo. Conserva lo stesso significato lessicale che ha anche il verbo dal quale si forma. II gerundio non ha la categoria del tempo, della persona e della diatesi; tutti i verbi al gerundio hanno significato attivo, inclusi quelli deponenti. A differenza del gerundivo, il gerundio non ha declinazioni plurali, si usa cioè soltanto al singolare.

A parte le forme che abbiamo menzionato, nei testi latini si trovano, con frequenza, le forme -undi, -undo, -undum, -undo del gerundio, chiamate forme antiquate. (Marco de Giorgi, Piero Mandressi, 1999: 43; Alfred Ernout, 1941: 275-6). Tali forme si usano e svolgono le stesse funzioni come le forme classiche del gerundio. Per esempio:

$>$ Vigilando, agundo, bene consulundo prospere omnia cedunt.

$\Leftrightarrow$ Duke vigjëluar, duke vepruar, duke gjykuar mirë, gjithçka ecën mbarë. (Sal. kap. LII).

$>$ Caesar dando, sublevando, ignoscundo, Cato nihil largiundo gloriam adeptus est.

$\leftrightarrow$ Çezari, duke dhënë, duke ndihmuar dhe duke falur, qe bërë i lavdishëm, ndërsa Katoni duke mos lejuar asgjë. (Sal. kap. LIV).

$>$ Sed serius a terra provectae naves neque usae nocturna aura in redeundo offenderunt.

$\Leftrightarrow$ Por anijet, të larguara pak më vonë nga toka, e pësuan keq gjatë rikthimit, duke mos përfituar dot nga puhia e natës. (Caes. Liber III, kap. 8).

$>$ Pedites vero tantummodo umeris ac summo pectore exstarent et cum altitudine aquae tum etiam rapiditate fluminis ad transeundum impedirentur.

$\Leftrightarrow$ Ndërsa këmbësorëve u qëndronin jashtë ujit vetëm shpatullat dhe pjesa e sipërme e kraharorit dhe pengoheshin të kalonin lumin jo vetëm nga thellësia e ujit, por edhe nga rryma. (Caes. Liber I, kap. 62).

\section{L'uso Nominale del Gerundio}

Il caso genitivo del gerundio si usa con alcuni sostantivi e aggettivi che richiedono generalmente il caso genitivo. Si usa:

a) Quando dipende dai sostantivi: ars, potestas, facultas, signum, occasio, locus (che indicano possibilità oppure occasione), initium, finis, voluntas ecc. (Tantucci, 1992: 368). Per esempio:

$>$...quorum vocibus et concursu terrentur infirmiores, dubii confirmantur, plerisque vero libere decernendi potestas eripitur.

$\stackrel{4}{\longrightarrow}$...nga tërsëllimi dhe ardhja e tyre tmerrohen senatorët frikacakë, marrin zemër të lëkundurit, por shumica nuk mundi të shfaqte mendimin lirshëm. (Caes. Liber I, kap. 3)

$>$ Quoniam ad id tempus facultas colloquendi non fuerit, atque ipse Brundisium sit venturus, interesse rei publicae et communis salutis se cum Pompeio colloqui. 
$\leftrightarrow$ Meqë deri në atë kohë nuk kishte qenë e mundur të bisedonin, dhe vetë Çezari po vinte në Brindizi, ishte në dobi të vendit dhe të të gjithëve që ai të bisedonte me Pompeun. (Caes. Liber I, kap. 24).

$>$ Caesar Brundisium ad suos severius scripsit, nacti idoneum ventum ne occasionem navigandi dimitterent, sive ad litora Apolloniatium cursum dirigere atque eo naves eicere possent.

$\stackrel{n}{\rightarrow}$ Çezari u shkroi mjaft rreptë njerëzve të tij në Brindizi, që sapo të kishte erë të mbarë, të mos humbisnin rastin për lundrim dhe të përpiqeshin të mbanin drejtimin e të bregëzonin anijet ose në brigjet e Apolonisë...(Caes. Liber III, kap. 25).

$>$ De cuius hominis moribus pauca prius explananda sunt, quam initium narrandi faciam.

$\stackrel{\leftrightarrow}{\rightarrow}$ Përpara se të nis fillimin e tregimit, më parë po shpjegoj me pak fjalë karakterin e këtij njeriu. (Sal. kap. IV).

$>$ Caesar etsi ad spem conficiendi negotii maxime probabat coactis navibus mare transire et Pompeium sequi,...

$\stackrel{\leftrightarrow}{\hookrightarrow}$ Çezari, me shpresën e përfundimit sa më parë të sipërmarrjes, gjykonte të nevojshme që të kalonte detin me anije dhe të ndiqte Pompeun,... (Caes. Liber I, kap. 29).

$>$ Ars vivendi - arti i të jetuarit (i jetës)

$>$ Signum pugnandi - shenja e të luftuarit (e luftës)

$>$ Spes vicendi - shpresa e të fituarit (e fitores)

$>$ Locus fugiendi - mundësia e të arratisurit (e arratisjes)

$>$ Nox finem pugnandi fecit. - Nata shënoi fundin e të luftuarit (e luftës) (Piazzino \& Fragonara, 1985: 420).

$>$ Ars scribendi librum - Arti i të shkruarit një libër. (Tantucci, 1992: 367)

Come si può notare dagli ultimi esempi, il gerundio al genitivo dipendente dai sostantivi soprammenzionati, si può tradurre in albanese con un sostantivo verbale. Mentre dagli esempi trovati in Cesare e Sallustio, grazie allo spazio permesso dalla traduzione, il gerundio possiamo tradurlo anche con un sostantivo non verbale. Esso dipende da un sostantivo 0 aggettivo e serve per completarli. In questi casi ha la funzione di un determinativo.

b) Quando dipende dagli aggettivi che indicano desiderio 0 abilità quali: cupidus, avidus, studiosus, peritus ecc.

Per esempio: (Piazzino \& Fragonara, 1985: 421)

$>$ Germani bellandi peritissimi erant. - Gjermanët ishin mjaft të regjur për luftë.

$>$ Imperator noster milites decertandi cupidos vix retinuit. - Gjenerali ynë mezi i mbajti ushtarët që donin të luftonin.

$>$ Discipulus diligens flagrat cupiditate legendi. - Nxënësi i zellshëm digjet nga dëshira për të lexuar.

c) Fra gli usi del gerundio del caso genitivo un'attenzione particolare merita l'uso con le parole causa o gratia che insieme al gerundio esprimono una proposizione finale (Terracina, 2003: 68). Una tale costruzione in albanese si traduce con l'infinito del tipo për të punuar con funzione finale. Per esempio:

> Postremo dissimulandi causa aut sui expurgandi, sicut iurgio lacessitus foret, in senatum venit.

$\stackrel{\leftrightarrow}{\rightarrow}$ Më në fund, për të ngatërruar ujërat ose për t'u shfajësuar, sikur po akuzohej padrejtësisht, shkoi në senat. (Sal. kap. XXXI).

$>$ Afranius Petreiusque terrendi causa atque operis impediendi copias suas ad infimas montis radices producunt et proelio lacessunt.

$\Leftrightarrow$ Afrani dhe Petrei për t'i frikësuar dhe për t'u penguar çezarianëve fortifikimin e kampit, i shtynë forcat e veta deri në rrëzë të malit dhe filluan të ngacmonin për luftë. (Caes. Liber I, kap. 42).

$>$ Has inter se capreolis molli fastigito coniungunt, ubi tigna, quae musculi tegendi causa ponant, collocentur.

$\stackrel{\leftrightarrow}{\hookrightarrow}$ Këto i bashkojnë mes tyre me këmbalecë me pjerrësi të lehtë, mbi të cilat mbështeten trarët për të mbuluar galerinë. (Caes. Liber II, kap. 10).

$>$ In curiam veni audiendi causa.

$\stackrel{\leftrightarrow}{\hookrightarrow}$ Erdha në mbledhje për të dëgjuar.

> Multi autem, qui e castris visundi aut spoliandi gratia processerant, volventes hostilia cadavera amicum alii, pars hospitem aut cognatum reperiebant.

$\stackrel{\leftrightarrow}{\hookrightarrow}$ Shumë përsona, që kishin dalë nga shtatorret, për të parë ose për të bërë plaçkë, duke kthyer mbarë kufomat e armiqve, kishin gjetur një shok, të tjerë një mik ose një të afërm. (Sal. kap. LXI).

L'uso del gerundio al genitivo con le parole gratia e causa in albanese si traduce sempre con la forma implicita del tipo për të punuar ed esprime scopo.

Il caso dativo del gerundio si usa raramente. Si usa come complemento di fine (dativus finalis) o di limitazione (dativus limitationis). Dipende di solito: 
a) Dagli aggettivi che indicano attitudine o propensione quali: idoneus, aptus, utilis, inutilis ecc. In questi casi il gerundio si può tradurre in albanese con un sostantivo oppure con la forma implicita del tipo për të punuar. Per esempio: (Terracina, 2003: 69)

$>$ Locus idoneus pugnando - vend $i$ përshtatshëm për luftim (për të luftuar).

$>$ Hora navigando apta - ora e përshtatshme për lundrim (për të lundruar).

$>$ Qui sibi imperare nescit ne aliis quidem praecipiendo aptus est -

$\Leftrightarrow$ Kush nuk di të komandojë veten, nuk është i përshtatshëm të komandojë as të tjerët.

$>$ Aqua utilis est bibendo - Uji është i dobishëm për pirje (për të pirë).

b) Dai verbi o dalle espressioni verbali che richiedono il caso dativo quali: adsum (jam pranë), intersum (jam në mes), praesum (jam në krye), sufficio (mjaftoj), satis sum (jam i mjaftueshëm), operam do (pëpiqem), diem status (fiksoj një ditë) ecc. Per esempio: (Piazzino \& Fragonara, 1985: 421)

$>$ Constabat Crassum scribendo adfuisse - Dihej që Krasi kishte qenë i pranishëm në regjistrim.

$>$ Interfui pugnando - mora pjesë në luftim; Operam do scribendo - përpiqem për të shkruar.

Generalmente il dativo del gerundio si usa raramente, poiché sostituito dall'accusativo con la preposizione ad (Ghiselli \& Concialini, 2006: 180), che in albanese nella maggioranza dei casi si traduce con la forma implicita del tipo për të punuar.

II caso accusativo del gerundio si incontra spesso e si usa sempre con la preposizione ad. Esso ha il significato di scopo e di solito si traduce con la forma implicita del tipo për të punuar oppure con il congiuntivo. II gerundio in questo caso ha la funzione di un complemento indiretto. Si usa: (Terracina, 2003: 69)

a) In dipendenza dagli aggettivi che indicano attitudine o propensione quali: aptus, idoneus, prospensus, utilis, inutilis. Per esempio:

$>$ Castra erant ad bellum ducendum aptissima natura loci et munitione et maris propinquitate et aquae et salis copia,...

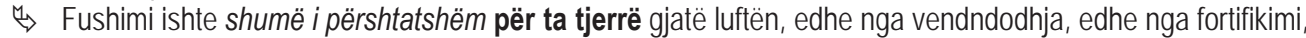
edhe nga afria me detin, edhe nga bollëku i ujit dhe i kripës... (Caes. Liber II, kap. 37).

$>$ Quadriremes omnes et quinqueremes aptae instructaeque omnibus rebus ad navigandum.

$\stackrel{\leftrightarrow}{\leftrightarrow}$ Të gjitha ishin katër dhe pesërremëshe, kishin përvojë të mirë dhe ishin të përshtatshme me gjithçka për të lundruar. (Caes. Liber III, kap. 111).

$>$...cetrati auxiliaresque nullam, quorum erant et facultates ad parandum exiguae et corpora insueta ad onera portanda.

$\stackrel{4}{4}$...cetratët dhe trupat ndihmëse nuk kishin fare, pasi kishin pak mundësi për të siguruar dhe nuk ishin mësuar për të mbajtur pesha. (Caes. Liber I, kap. 78).

$>$ Non solun ad discendum propensi simus, sed etiam ad docendum.

$\Leftrightarrow$ Jemi të prirur jo vetëm për t'u mësuar, por edhe për të mësuar.

$>$ Magistratus ad duas res diversissimas sint apti, ad oboediendum atque ad imperandum.

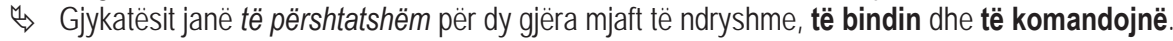

b) Per esprimere una proposizione finale. Per esempio:

$>$...hortaturque eos, ne animo deficiant quaeque usui ad defendendum oppidum sint parent.

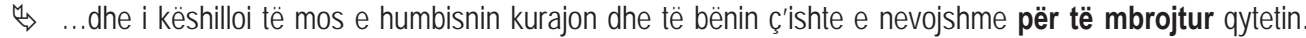
(Caes. Liber I, kap. 19).

$>$ Ibique naves expediunt rursusque se ad confligendum animo confirmant et consilia communicant.

$\stackrel{\leftrightarrow}{\hookrightarrow}$ Dhe aty përgatisin anijet, marrin sërish zemër për të luftuar dhe shkëmbejnë mendime për planin e sulmit. (Caes. Liber II, kap. 4).

$>$ Fuere ea tempestate, qui dicerent Catilinam oratione habita, cum ad ius iurandum popularis sceleris sui adigeret, ...

$\stackrel{n}{\hookrightarrow}$ Në atë kohë pati njerëz që thoshin se Katilina, si mbajti fjalimin, i detyronte shokët e komplotit që të betoheshin,... (Sal. kap. XXII).

$>$ Neque illi tamen ad cavendum dolus aut astutiae deerant.

$\Leftrightarrow$ Këtij nuk i mungonte dredhia apo mendjemprehtësia për t'u mbrojtur. (Sal. kap. XXVI).

\section{II Caso Ablativo del Gerundio si Può Usare con o Senza Preposizione}

a) Nel caso ablativo il gerundio viene preceduto dalle preposizoni ab, in, de, ex, ecc. In questi casi, così come il sostantivo accompagnato dalla preposizione, il gerundio acquisisce le sue particolarità. Sotto l'influenza della 
preposizione il gerundio si mette nel caso ablativo e svolge la funzione di un complemento (Terracina, 2003: 70). Se non ci fosse la preposizione, il gerundio si metterebbe all'accusativo. Con la preposizione in il gerundio in questo caso si traduce di solito con il gerundio albanese, mentre quando si usa con de e ex si può tradurre con un sostantivo. Per esempio:

$>$ Ita tuto ac sine ullo vulnere ac periculo sex tabulata exstruxerunt fenestrasque, quibus in locis visum est, ad tormenta mittenda in struendo reliquerunt.

$\stackrel{\leftrightarrow}{\hookrightarrow}$ Kështu, në siguri të plotë, pa asnjë plagë e rrezik, ngritën kullën me gjashtë kate dhe lanë frëngji, nga të cila shikoje vendin, duke ngritur makina hedhëse (gurësh e shigjetash). (Caes. Liber II, kap. 9).

$>\mathrm{Ne}$ aut ingratus in referenda gratia aut arrogans in praeripiendo populi beneficio videretur.

$\Leftrightarrow$ Kështu, ai nuk bëhej bukëshkalë për shërbimin e ofruar, as mendjemadh duke nëpërkëmbur popullin për të mirën që i takonte vetëm atij. (Caes. Liber III, kap. 1).

$>$ Sed praeoccupatus animus Attianorum militum timore et fuga et caede suorum nihil de resistendo cogitabat,...

$\stackrel{\leftrightarrow}{\leftrightarrow}$ Por, ushtarët e Acit, të pushtuar nga tmerri, nga arratia dhe nga gjakderdhja e shokëve, as nuk e mendonin përballjen me armikun,... (Caes. Liber II, kap. 34).

> Ex providendo est appellata prudentia. - Prudentia (maturia, zgjuarsia) është quajtur kështu nga parashikimi (providēre). (Piazzino \& Fragonara, 1985: 422)

Il gerundio al caso ablativo svolge la funzione del complemento quando si usa specialmente con la preposizione de, la quale in albanese si traduce con un sostantivo. Mentre negli altri casi svolge la funzione di un complemento.

b) Senza preposizione il gerundio all'ablativo ha la funzione di un ablativo di mezzo, maniera e, in casi sporadici, di causa (Tantucci, 1992: 369). In tali casi il gerundio si traduce in albanese con l'infinito. Per esempio:

$>$ Neque vero coniuncti Albici comminus pugnando deficiebant neque multum cedebant virtute nostris.

$\Leftrightarrow$ Madje, të bashkuar me albikët, nuk u shmangën, duke luftuar fytafyt dhe, për nga trimëria, nuk binin më poshtë se tanët. (Caes. Liber II, kap. 6).

$>$ Namque a principio consulatus sui multa pollicendo per Fulviam effecerat, ut Q. Curius, de quo paulo ante memoravi, consilia Catilinae sibi proderet.

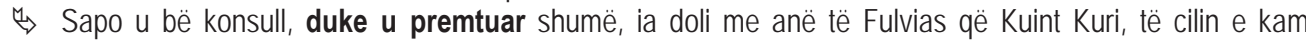
përmendur pak më lart, t'i tregonte hollësisht planet e Katilinës. (Sal. kap. XXVI).

$>$ Nocturnis consiliis armorum atque telorum portationibus, festinando, agitando omnia plus timoris quam periculi effecerant.

$\Leftrightarrow$ Bënin takime nate dhe transportime armësh, duke i nxitur dhe tubulluar të gjithë, duke shkaktuar më tepër frikë sesa rrezik. (Sal. kap. XLI).

$>$ Illos dubitando et dies prolatando magnas opportunitates corrumpere.

$\stackrel{\leftrightarrow}{\leftrightarrow}$ Ata, duke dyshuar dhe duke shtyrë ditët, u shkonin kot mjaft raste të volitshme. (Sal. kap. XLIII).

Da tutti i casi del gerundio, soltanto il caso ablativo si può tradurre con il gerundio dell'albanese. La funzione di modo e causa che il gerundio ha nel caso ablativo lo troviamo anche nel gerundio albanese.

\section{L'uso Verbale del Gerundio}

L'uso verbale del gerundio si collega alle caratteristiche sintattiche che ha acquisito. La capacità di reggere complemento diretto e di essere determinato da un avverbio sono caratteristiche che lo relazionano al sistema verbale, così come il gerundio dell'albanese. La capacità di reggere un complemento diretto è la caratteristica principale per dare la definizione del verbo ad una parola. In questi casi il gerundio segue il complemento diretto, ma in alcuni testi lo abbiamo visto anche collocato davanti. Quando il gerundio è all'accusativo, il complemento di solito si mette tra le parti costituenti; la preposizione ad ed il gerundio (Mckeown, 2010: 236). Mentre quando viene completato da un avverbio, quest'ultimo di solito precede il gerundio. Notiamo i seguenti casi:

\section{L'uso con il Complemento Diretto}

Gen. Ars carmina cantandi difficilis est. - Arti i të kënduarit të këngës është i vështirë.

Dat. Carmina cantando operam dedit. - U përpoq të këndonte një këngë.

Acc. Cum amicis ad carmina cantandum abiit. - Me miqtë shkoi për të kënduar një këngë.

Abl. Carmina cantando uxori placuit. - Duke kënduar një këngë, kënaqi të shoqen. 
> ...praesidiis enim dispositis omnia litora a Caesare tenebantur, neque lignandi atque aquandi neque naves ad terram religandi potestas fiebat.

$\leftrightarrow$...Çezari kontrollonte tërë zonën bregdetare me garnizone të vogla, prandaj kundërshtari nuk kishte mundësi të siguronte dru e ujë dhe as të lidhte anijet në tokë. (Caes. Liber III, kap. 15).

> Qui omnes ad eum producti contra religionem iurisiurandi in eius conspectu crudelissime interficiuntur.

$\leftrightarrow$ Të gjithë këta çohen tek ai dhe, me gjithë fjalën e dhënë, i vret mizorisht para syve të vet. (Caes. Liber III, kap. 28).

$>$ Homines adulescentes summam potestatem nacti, quibus aetas animusque ferox erat, coepere senatum criminando plebem exagitare, dein largiundo atque pollicitando magis incendere, ita ipsi clari potentesque fieri.

$\Leftrightarrow$ Disa të rinj, që u gjendën rastësisht, me moshë dhe me shpirt të papërmbajtur, filluan të ngrinin peshë popullin, duke akuzuar senatin, më pas filluan të miklonin më tepër shpirtin e tij, duke dhuruar e premtuar, dhe kështu u bënë të njohur dhe të rëndësishëm. (Sal. kap. XXVIII).

$>$ Illos dubitando et dies prolatando magnas opportunitates corrumpere.

$\stackrel{\leftrightarrow}{\rightarrow}$ Ata, duke dyshuar dhe duke shtyrë ditët, u shkonin kot mjaft raste të volitshme. (Sal. kap. XLIII).

$>$...hortaturque eos, ne animo deficiant quaeque usui ad defendendum oppidum sint parent.

$\leftrightarrow$...dhe i këshilloi të mos e humbisnin kurajon dhe të bënin ç'ishte e nevojshme për të mbrojtur qytetin. (Caes. Liber I, kap. 19).

$>$ Scipio ad sequendum paratus equitum magnam partem ad explorandum iter Domitii et cognoscendum praemisit.

$\leftrightarrow$ Skipioni, i përgatitur për ta ndjekur, nisi përpara një pjesë të madhe të kalorësisë, për të zbuluar dhe për të njohur rrugën e Domicit. (Caes. Liber III, kap. 38).

$>$ Pompeius quoque de Caesaris consilio coniectura iudicans ad Scipionem properandum sibi existimabat.

$\Leftrightarrow$ Pompeu, duke marrë me mend planin e Çezarit, mendoi që të nxitonte te Skipioni. (Caes. Liber III, kap. 78).

$>$ Ea res in primis studia hominum accendit ad consulatum mandandum M. Tullio Ciceroni.

$\stackrel{\mapsto}{\rightarrow}$ Kjo gjë i nxiti njerëzit për t'ia dhënë konsullatin M. T. Cicëronit. (Sal. kap. XXIII).

\section{Completamento con L'avverbio}

Neque vero coniuncti Albici comminus pugnando deficiebant neque multum cedebant virtute nostris.

$\Leftrightarrow$ Madje, të bashkuar me albikët, nuk u shmangën, duke luftuar fytafyt dhe, për nga trimëria, nuk binin më poshtë se tanët. (Caes. Liber II, kap. 6).

$>$ Vigilando, agundo, bene consulundo prospere omnia cedunt.

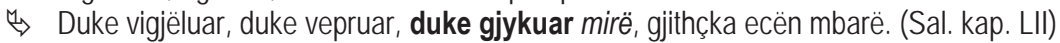

$>$ Nemo erat adeo tardus aut fugiens laboris, quin statim castris exeundum atque occurrendum putaret.

$\Leftrightarrow$ Sigurisht, asnjëri nuk ishte vonë dhe i bëri bisht punës, sa të mos mendonte që të dilte menjëherë nga kampi dhe të paraprinte. (Caes. Liber I, kap. 69).

$>$ Postremo dissimulandi causa aut sui expurgandi, sicut iurgio lacessitus foret, in senatum venit.

$\stackrel{\hookrightarrow}{\hookrightarrow}$ Më në fund për t'u fshehur ose për t'u shfajësuar, sa për të ngatërruar ujërat, shkoi në senat. (Sal. kap. $X X X I)$.

Come abbiamo già detto il gerundio ha significato attivo e per esprimere il significato passivo il latino usa il pronome riflessivo se ed il pronome ipsum. La caratteristica che allontana il gerundio dal verbo, nei testi e le grammatiche a cui abbiamo fatto riferimento, è l'impossibilità di essere usato con la particella negativa mos (non).

\section{Conclusioni}

II gerundio latino è un sostantivo verbale che designa un'azione in quanto processo. A differenza dell'infinito dell'albanese, esso viene costruito in modo sintetico aggiungendo al tema del presente i suffissi $-n d$, per i verbi di prima e seconda coniugazione, ed -end per i verbi di terza e quarta coniugazione. II gerundio ha caratteristiche nominali e verbali così come l'infinito latino. Esso si usa solo nella forma attiva e si può formare quasi in tutti i verbi attivi 0 deponenti, transitivi e intransitivi. II gerundio non ha la categoria del tempo, ma ha solo quattro casi (genitivo, dativo, accusativo e ablativo). Nel latino il gerundio si avvicina semanticamente con l'infinito, poiché si vede come complemento del sistema dei casi di quest'ultima quando si usa nella funzione di un sostantivo. II gerundio conserva lo stesso significato lessicale che ha anche il verbo dal quale si forma. Esso si usa solamente nel singolare. 
II gerundio nel caso genitivo, dipendente dai sostantivi che abbiamo menzionato, si può tradurre in albanese con un sostantivo verbale e raramente con un sostantivo non verbale. In questi casi esso assume la funzione del determinativo. Con le parole causa o gratia che insieme al gerundio esprimono una proposizione finale, si traduce in albanese sempre con una forma implicita del tipo për të punuar.

Nel caso dativo il gerundio si usa molto raramente e svolge la funzione di un complemento di fine (dativus finalis) 0 di limitazione (dativus limitationis). In casi simili il gerundio si può tradurre con un sostantivo oppure con la forma implicita del tipo për të punuar. Nel caso accusativo il gerundio si incontra spesso e si usa sempre con la preposizione ad. Esso ha il significato di scopo e di solito si traduce con la forma implicita del tipo për të punuar. Il gerundio in questo caso ha la funzione di un complemento indiretto. Nel caso ablativo il gerundio si può usare con o senza preposizione. Viene preceduto dalle preposizioni $a b$, in, de, ex, ecc., si mette nell'ablativo e svolge la funzione di un complemento. Con la preposizione in il gerundio in questo casi si traduce di solito con il gerundio albanese. Di tutti i casi del gerundio, soltanto quando è all'ablativo si può tradurre con il gerundio albanese. La funzione di modo e di causa che ha il gerundio all'ablativo senza preposizioone lo incontriamo anche nel gerundio dell'albanese. La capacità di reggere un complemento diretto e di essere determinato da un avverbio sono caratteristiche che collegano il gerundio con il sistema verbale. Quando accompagnato da un complemento, il gerundio di solito si colloca dopo quest'ultimo. Quando il gerundio è all'accusativo, il complemento si mette di solito tra le parti costituenti: la preposizione ad ed il gerundio. Mentre quando viene accompagnato da un avverbio, quest'ultimo di solito precede il gerundio.

\section{References}

Basha Nermin (1974), Përcjellorja e shqipes dhe gerundi e participi i latinishtes, punim diplome, Tiranë.

Caesar Caius Julius (2008), De bello civili, Torino: UTET.

Çeliku Mehmet (2006), Format e pashtjelluara të foljes në gjuhën e sotme shqipe, Tiranë: Shblu.

De Giorgi Marco, Mandressi Piero (1999), I verbi latini, Milano: Hoepli.

Ernout Alfred (1941), Morphologie historique du latin, Parigi: Librairie C. Klincksieck.

Ernous Alfred, Thomas François (1964), Syntaxe latine, Parigi: Klincksieck.

Famerie Etienne, Bodson Arthur, Dubuisson Michel (2012), Methode de langue latine, Parigi: Armand Colin.

Ghiselli Alfredo, Concialini Gabriella (2006), II nuovo libro di latino, Bari: Laterza.

McKeown Jc., (2010), Classical Latin, Indianapolis: Hackett Publishing.

Piazzino C., Fragonara A. (1985), Humanitatis magistra, Torino: Paravia.

Qiriazati Kosta, Dishnica Roza (1989), Gjuha latine, II, Tiranë: Shblu.

Sallustius Caius Crispus, (2008), Catilinae coniuratio, Milano: Oscarmondadori.

Tantucci Vittorio (1992), Urbis et orbis lingua, Bologna: Poseidonia.

Terracina Francesco (2003), Verbi latini, Tutti i verbi regolari e irregolari, Milano: Vallardi.

Xhamani Leonard (2012), Format e pashtjelluara të latinishtes dhe të shqipes (Vështrim krahasues), tesi di dottorato di ricerca. 